\title{
Palinologia do componente herbáceo na atmosfera de Caxias do Sul, RS, Brasil ${ }^{1}$
}

\author{
Sandra Maria Vergamini' ${ }^{2,5}$, Rosa Maria Valencia-Barrera ${ }^{3}$, Francieli Sbersi ${ }^{4}$ e Taísa Fedrizzi Maffazzioli ${ }^{4}$
}

\author{
Recebido em 3/01/2006. Aceito em 20/06/2006
}

\begin{abstract}
RESUMO - (Palinologia do componente herbáceo na atmosfera de Caxias do Sul, RS, Brasil). Este estudo foi realizado na cidade de Caxias do Sul de 1/janeiro/2001 a 31/dezembro/2002 e objetivou identificar os tipos polínicos de táxons herbáceos presentes na atmosfera e suas oscilações sazonais. A cidade está localizada na Encosta Superior Nordeste, Estado do Rio Grande do Sul. Seu clima é tropical pluvisazonal e apresenta três diferentes tipos de vegetação: Floresta Ombrófila Mista, Floresta Decidual e Campo. O monitoramento polínico foi realizado utilizando o captador volumétrico de sucção Burkard®, instalado no topo do Hospital Geral a uma altura aproximada de $20 \mathrm{~m}$. Durante o período de estudo foram identificados 40 tipos polínicos, sendo 11 de táxons herbáceos, representando 23,22\% do total de grãos coletados. A concentração máxima das táxons herbáceos registrou-se nos meses de outubro e novembro. O tipo polínico Poaceae R.Brown apresentou a maior incidência, seguido por Cyperaceae Juss., Plantago L., Amaranthus L., Rumex L., Iridaceae Juss., Brassicaceae Burnett, Apiaceae Lindl., Alternanthera Forssk., Convolvulaceae Juss. e Caryophyllaceae Juss.
\end{abstract}

Palavras-chave: Aeropalinologia, pólen, táxons herbáceos, Burkard®, Caxias do Sul

\begin{abstract}
Palinology of the herbaceous component in the atmosphere of Caxias do Sul, Rio Grande do Sul State, Brazil). This study was carried out in the city of Caxias do Sul, from January 1, 2001 to December 31, 2002 and aimed to identify pollen types and seasonal fluctuation of herbaceous taxa in the atmosphere. The city is located on the Upper Northeast Slopes in Rio Grande do Sul State. The climate is tropical with seasonal rainfall and there are three different types of vegetation: mixed ombrophilous forest, deciduous forest and grassland. Pollen monitoring was done with a Burkard $®$ volumetric spore-trap sampler placed on top of the Hospital Geral, about $20 \mathrm{~m}$ above ground level. During the study period 40 pollen types were identified, 11 from herbaceous taxa, which represents $23.22 \%$ of the total number of grains collected. Maximum concentration of herbaceous taxa was registered in October and November. The Poaceae R.Brown pollen type had the highest incidence, followed by Cyperaceae Juss., Plantago L., Amaranthus L., Rumex L., Iridaceae Juss., Brassicaceae Burnett, Apiaceae Lindl., Alternanthera Forssk., Convolvulaceae Juss. and Caryophyllaceae Juss.
\end{abstract}

Key words: Aeropalinology, pollen, herbaceous taxa, Burkard®, Caxias do Sul

\section{Introdução}

O termo Aerobiologia foi conhecido nos anos 30, por Fred C. Meier, com a finalidade de incluir essa denominação para os estudos em realização sobre esporos de fungos, grãos de pólen e bactérias encontrados na atmosfera (Gregory 1973). O estudo da Aerobiologia apresenta dificuldades, em parte por ser uma ciência jovem que está em constante evolução e devido às diferentes perspectivas de aplicação desta ciência em diversos campos. Cada uma de suas especialidades é interdisciplinar, necessitando da cooperação de outras áreas do conhecimento (agronomia, medicina, física, botânica, zoologia, ecologia, etc.). Compreende a liberação, retenção, dispersão, deposição e incidência atmosférica de grãos de pólen, esporos e microrganismos aerotransportados (Pathirane 1975). Na atmosfera encontram-se grãos de pólen que flutuam e estão sujeitos à gravidade, ao atrito com as moléculas gasosas que as rodeiam e a fatores climáticos (direção e velocidade do vento, precipitação, temperatura e umidade) decisivos para sua dispersão e permanência na atmosfera. Os grãos de pólen possuem morfologia característica, possibilitando a identificação de táxons quanto ao tamanho, forma, à superfície, às aberturas, à simetria e polaridade.

No Brasil, trabalhos sobre Aeropalinologia foram realizados com o uso de diferentes metodologias e períodos de amostragem (Oliveira

\footnotetext{
Parte da Tese de Doutorado da primeira Autora

2 Universidade de Caxias do Sul, Museu de Ciências Naturais, C. Postal 1352, 95020-972 Caxias do Sul, RS, Brasil

3 Universidad de León, Departamento de Biologia Vegetal, Campus de Vegazana, 24071 León

4 Universidade de Caxias do Sul, Acadêmica de Ciências Biológicas, Bolsista

5 Autor para correspondência: smvergam@ucs.br
} 
Lima \& Greco 1942a; Oliveira Lima \& Greco 1942b; Oliveira Lima \& Guimarães 1958; Salgado-Labouriau 1973; Rosário Filho 1983; Lorscheitter \& Bernd 1990; Hilgert 1998).

O objetivo do presente trabalho é o de identificar e quantificar os diferentes tipos polínicos de plantas herbáceas ocorrentes na atmosfera de Caxias do Sul e suas oscilações sazonais.

\section{Material e métodos}

Caracterização da área de estudo - A cidade de Caxias do Sul está localizada na Encosta Superior do Nordeste do Estado do Rio Grande do Sul, entre os meridianos $51^{\circ} 30^{\prime}$ e $51^{\circ} 00^{\prime}$ Oeste e os paralelos $28^{\circ} 15^{\prime}$ e $29^{\circ} 30^{\prime}$ Sul (Fig. 1). A altitude da área de estudo varia entre o máximo de 760 metros e um mínimo de 100 metros junto ao Rio Caí, e apresenta uma área física de $1.657 \mathrm{~km}^{2}$. A população é de aproximadamente 396.261 habitantes.

O município caracteriza-se pela ocorrência de três formações vegetais distintas: a Floresta Ombrófila Mista, a Savana-Gramíneo Lenhosa e a Floresta Estacional Decidual. A Floresta Ombrófila Mista, conhecida como Mata de Araucária, é a formação mais extensa da região em estudo. O clima é mesotropical, com quatro estações definidas. A pluviosidade média na cidade é de $1.821 \mathrm{~mm} /$ ano e a temperatura média é de $16^{\circ} \mathrm{C}$. A umidade relativa do ar varia de 75 a $85 \%$.

Coleta e análise de dados - O monitoramento polínico diário realizou-se de 1/janeiro/2001 a 31/dezembro/2002, utilizando o captador volumétrico de sucção tipo Hirst (Burkard®). O captador está instalado no topo do Hospital Geral, em Caxias do Sul, a uma altura aproximada de 20 metros, nas coordenadas 22J0484862

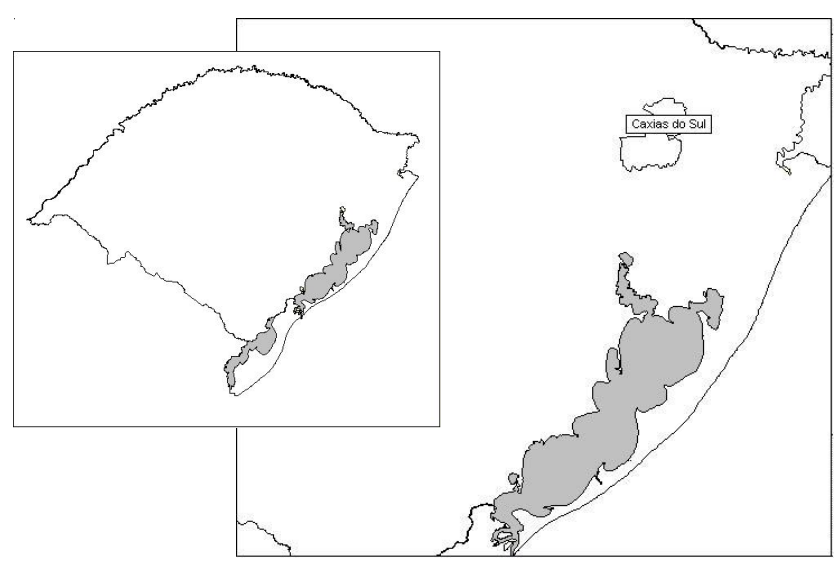

Figura 1. Localização da área de estudo.
UTM 6774144, não havendo obstáculos que bloqueiem a chegada de massas de ar de nenhuma direção (Fig. 2).

O captador manteve uma entrada de ar constante de $10 \mathrm{~L} / \mathrm{min}$ que incidiu sobre uma superfície de captura (fita de melinex) impregnada com silicone, na qual se fixaram as partículas aéreas, o que inclui os grãos de pólen. Essa fita, trocada semanalmente, foi cortada em sete partes, que corresponderam aos sete dias da semana. Após, as lâminas foram confeccionadas utilizando-se gelatina glicerinada com fucsina e analisadas.

As análises foram realizadas com um microscópio ótico Leica DMLS, sendo a leitura das mesmas de acordo com o postulado de Domínguez Vilches et al. (1991), realizando-se quatro varreduras longitudinais eqüidistantes, utilizando uma objetiva de aumento 40x. As concentrações polínicas foram expressas em grãos $/ \mathrm{m}^{3}$ de ar. A metodologia utilizada neste trabalho é inédita no Brasil.

As identificações polínicas basearam-se numa coleção de referência, a palinoteca, confeccionada com material fresco ou herborizado da região, além de atlas especializados, como Hyde \& Adams (1958), Grant Smith (1986), Valdés et al. (1987), Moore et al. (1991), Pire et al. (1998).

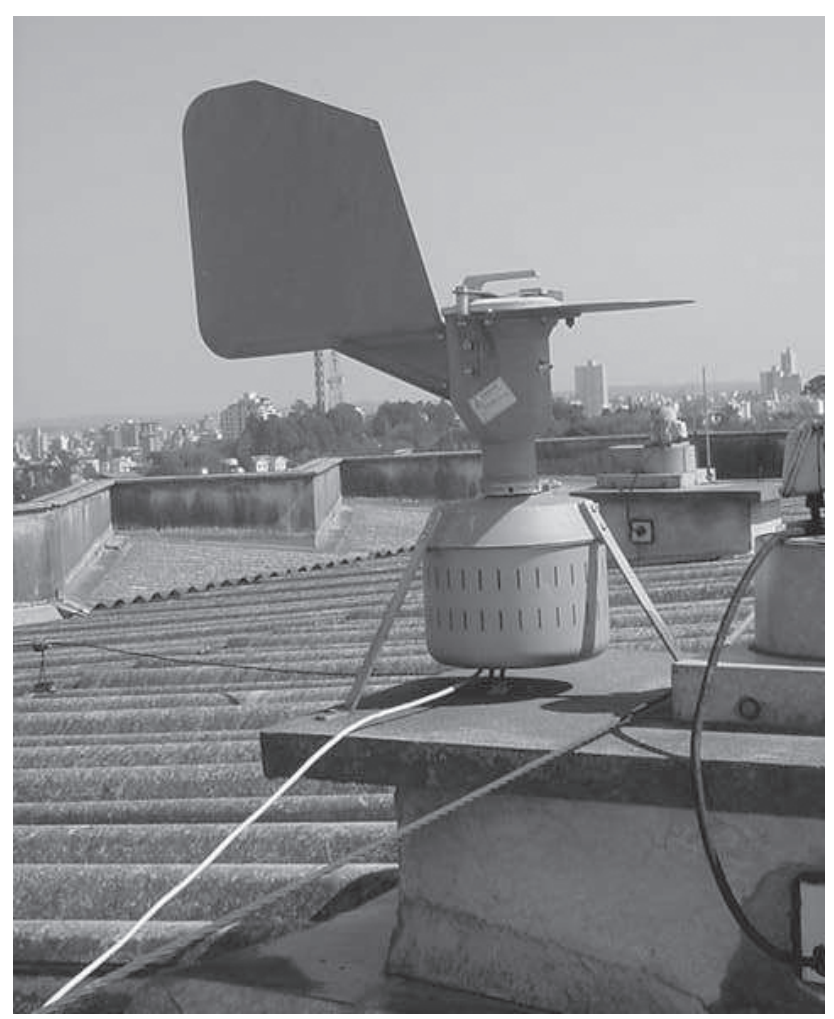

Figura 2. Coletor Burkard Manufacturing®®. 


\section{Resultados}

Foram identificados, durante o período de estudo, 40 tipos polínicos na atmosfera de Caxias do Sul, num total de 30.469 grãos de pólen. Em 2001 foram coletados 16.221 grãos de pólen, sendo 4.001 provenientes de plantas herbáceas, o que correspondeu a 24,66\% do total anual. Já em 2002, 14.248 grãos de pólen foram coletados, sendo 3.075 de plantas herbáceas $(21,58 \%)$.

Entre os 40 tipos polínicos identificados, 11 pertencem a plantas herbáceas de Poaceae R. Brown, Cyperaceae Juss., Plantago L. (Plantaginaceae), Amaranthus L. (Amaranthaceae), Rumex L. (Poligonaceae), Iridaceae Juss., Brassicaceae Burnett, Apiaceae Lindl., Alternanthera Forssk. (Amaranthaceae), Convolvulaceae Juss. e Caryophyllaceae Juss., em ordem de incidência. O total de pólen herbáceo correspondeu a 23,22\% do pólen contabilizado, com destaque para o tipo polínico Poaceae, que foi responsável por $19,07 \%$, ou $82,13 \%$ do total de pólen de plantas herbáceas no período estudado. Os outros 10 tipos polínicos representaram $4,15 \%$ do total de pólen coletado ou $17,87 \%$ de plantas herbáceas. Tanto em 2001 quanto em 2002, Poaceae R.Brown. foi o segundo tipo polínico mais incidente, representando $20,15 \%$ do total no primeiro ano e $17,85 \%$ no segundo. Entretanto, na soma total do período 2001-2002, foi o principal tipo polínico, correspondendo a $19,07 \%$.

Os grãos de pólen de plantas herbáceas encontram-se na atmosfera da cidade principalmente nos meses de primavera até o início do verão, sendo outubro e novembro os meses de maior incidência. Nos dois anos de estudo, as concentrações máximas mensais dos tipos polínicos deste grupo totalizaram 1.509 grãos de pólen $/ \mathrm{m}^{3}$ em outubro, 1.681 grãos de pólen $/ \mathrm{m}^{3}$ em novembro e 1.212 grãos de pólen $/ \mathrm{m}^{3} \mathrm{em}$ dezembro (Fig. 3).

No ano de 2001 encontrou-se, no mês de outubro, um total de 961 grãos $/ \mathrm{m}^{3}$ de plantas herbáceas, sendo que Poaceae R.Brown ( 839 grãos $/ \mathrm{m}^{3}$ ) foi o tipo polínico de maior incidência, seguido por Cyperaceae Juss. (50 grãos $/ \mathrm{m}^{3}$ ) e Poligonaceae - Rumex L. (33 grãos $/ \mathrm{m}^{3}$ ). Em novembro registrou-se a maior concentração, com um total de 1.058 grãos $/ \mathrm{m}^{3}$, sendo a maior quantidade de Poaceae R. Brown (949 grãos $/ \mathrm{m}^{3}$ ), seguido por Cyperaceae Juss. (69 grãos $/ \mathrm{m}^{3}$ ) e Plantago L. (31 grãos $/ \mathrm{m}^{3}$ ). No mês de dezembro registrou-se um total de 816 grãos $/ \mathrm{m}^{3}$, sendo Poaceae R. Brown (666 grãos $/ \mathrm{m}^{3}$ ), Cyperaceae Juss.

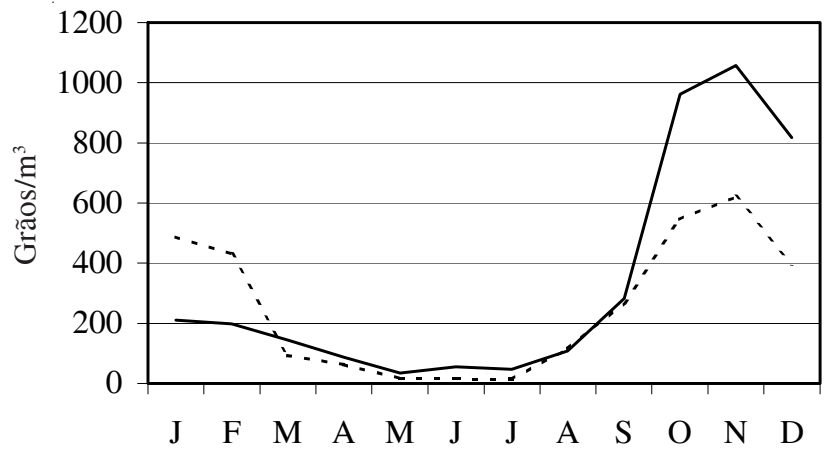

Figura 3. Evolução mensal da concentração atmosférica de táxons herbáceos durante os anos de 2001 (-) e 2002 (----).

(61 grãos $/ \mathrm{m}^{3}$ ) e Plantago L. (25 grãos $/ \mathrm{m}^{3}$ ) os mais incidentes (Fig. 4).

Em 2002, encontrou-se, no mês de outubro, um total de 548 grãos $/ \mathrm{m}^{3}$ de plantas herbáceas, sendo Poaceae R. Brown (485 grãos $/ \mathrm{m}^{3}$ ), Plantago L. (24 grãos $/ \mathrm{m}^{3}$ ) e Rumex L. (18 grãos $/ \mathrm{m}^{3}$ ) os tipos polínicos mais incidentes. No mês de novembro, registrou-se um total de 623 grãos $/ \mathrm{m}^{3}$, sendo Poaceae R. Brown (512 grãos $\left./ \mathrm{m}^{3}\right)$, Cyperaceae Juss. (65 grãos $/ \mathrm{m}^{3}$ ) e Rumex L. (17 grãos $/ \mathrm{m}^{3}$ ) os tipos polínicos que apresentaram as maiores concentrações. Em dezembro, registrou-se um total de 396 grãos $/ \mathrm{m}^{3}$, sendo Poaceae R.Brown (271 grãos $/ \mathrm{m}^{3}$ ), Cyperaceae Juss. (66 grãos $/ \mathrm{m}^{3}$ ) e Plantago L. $\left(23\right.$ grãos $\left./ \mathrm{m}^{3}\right)$ os tipos polínicos mais incidentes.

Na soma total do período 2001-2002, a ordem de incidência polínica de plantas herbáceas foi de Poaceae R. Brown (5.812 grãos $/ \mathrm{m}^{3}$ ), Cyperaceae Juss. (630 grãos $/ \mathrm{m}^{3}$ ), Plantaginaceae - Plantago L. (201 grãos $/ \mathrm{m}^{3}$ ), Amaranthaceae - Amaranthus L. (139 grãos $/ \mathrm{m}^{3}$ ), (Poligonaceae) Rumex L. (138 grãos $/ \mathrm{m}^{3}$ ) e, de forma esporádica, Iridaceae Juss. (76 grãos $/ \mathrm{m}^{3}$ ), Brassicaceae Burnett (42 grãos $/ \mathrm{m}^{3}$ ), Apiaceae Lindl. (20 grãos $/ \mathrm{m}^{3}$ ), Amaranthaceae Alternanthera Forssk. (9 grãos $/ \mathrm{m}^{3}$ ), Convolvulaceae Juss. (6 grãos $/ \mathrm{m}^{3}$ ) e Caryophyllaceae Juss. $\left(3\right.$ grãos $\left./ \mathrm{m}^{3}\right)$.

\section{Discussão}

Os grãos de pólen de táxons herbáceos foram encontrados na atmosfera de Caxias do Sul principalmente nos meses de novembro a janeiro, época de primavera e início de verão. A maior concentração ocorreu no mês de novembro. Poaceae foi o tipo polínico mais incidente na atmosfera da cidade.

Resultados semelhantes, quanto à época de dispersão polínica também foram encontrados na cidade 
de Buenos Aires, Argentina, por Noetinger (1993) no monitoramento de três anos pelo método Tauber; o pólen herbáceo foi encontrado nos meses de novembro a abril. Os primeiros estudos do conteúdo polínico da atmosfera da cidade de La Plata, Argentina (Nitiu \& Romero 2001), utilizando aparelho tipo Hirst, apresentaram resultados que, em sua maioria, corroboram com os obtidos na cidade de Caxias do Sul. O pólen de táxons herbáceos foi encontrado principalmente nos meses de outubro e novembro, época primaveril de floração da maioria das espécies desses tipos polínicos. Resultados análogos também foram registrados na cidade de Mar del Plata, por Pérez \& Paez (1998) quando as concentrações apresentaram sazonalidade e variações anuais. O período de dispersão polínica de táxons herbáceos começou na primavera tardia e no verão antecipado.
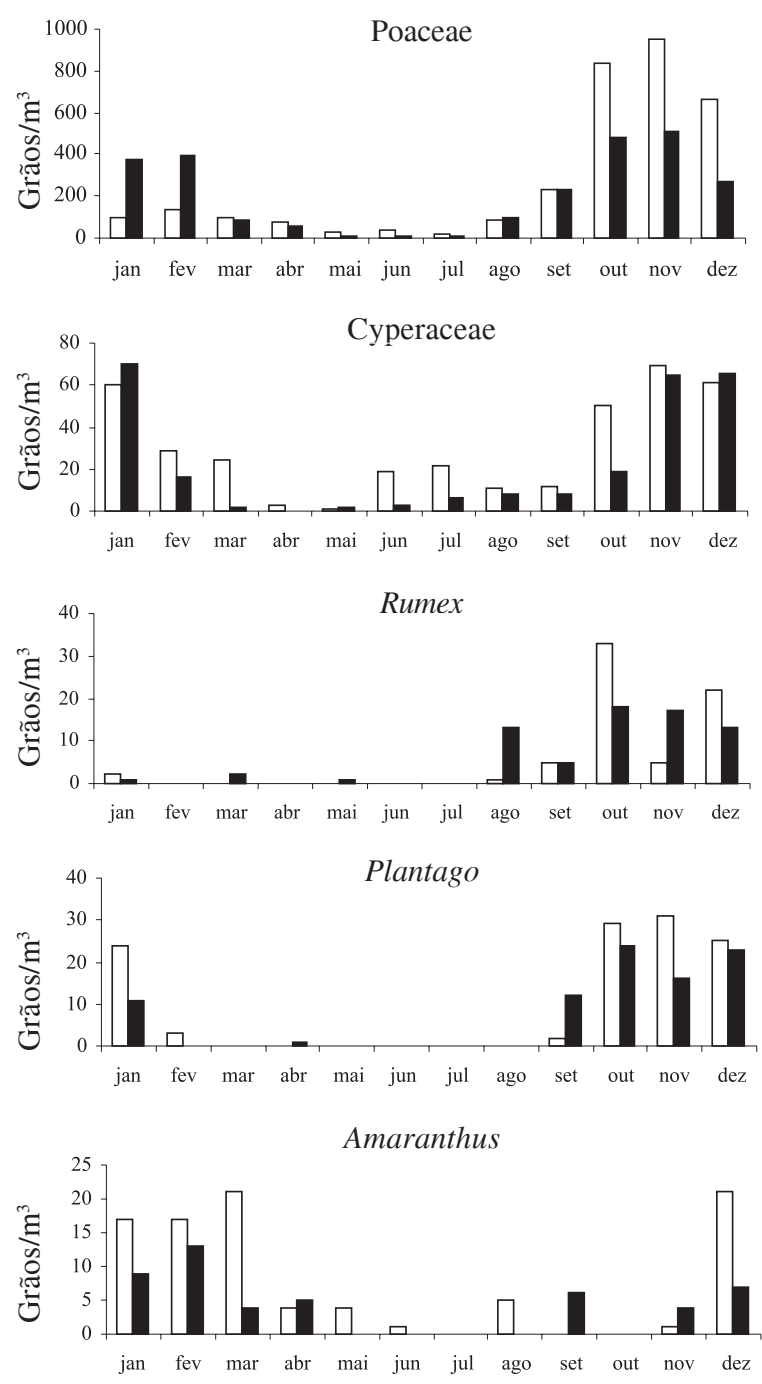

Figura 4. Concentrações mensais dos tipos polínicos de plantas herbáceas de Caxias do Sul durante os anos de 2001 (口) e 2002 (ロ).
A família Poaceae R. Brown representa o primeiro fator etiológico de alergia polínica do mundo e, na cidade de Caxias do Sul não é exceção (Vieira \& Negreiros 1989), pois os valores das concentrações desse tipo polínico foram os mais elevados em relação aos demais tipos na chuva polínica dessa cidade. Houve uma maior concentração deste tipo polínico no ano de 2001, sendo o pico mensal no mês de novembro nos dois anos de estudo.

O pólen de Cyperaceae Juss. apareceu na atmosfera da região estudada durante todo o ano e com maior intensidade no período primaveril e início de verão. O pico mensal ocorreu em novembro/2001 e em janeiro/2002. Resultados similares foram encontrados por Hilgert (1998). Nas cidades de Buenos Aires (Noetinger \& Romero 1997), La Plata (Nitiu \& Romero 2001) e Mar del Plata (Latorre \& Pérez 1997) o período de dispersão polínica coincidiu com este estudo.

A concentração de Plantago L. na atmosfera de Caxias do Sul foi bastante semelhante nos dois anos de estudo. Aparece na atmosfera da região estudada durante o período de primavera e verão. Os resultados deste estudo coincidem quanto à época de dispersão polínica e à quantidade de pólen encontrada por Hilgert (1998). No período primaveril, também foram encontrados grãos de pólen de Plantago em várias cidades da Argentina, como em Buenos Aires (Majas \& Romero 1992; Noetinger 1993), em Mar del Plata (Latorre \& Pérez 1997; Pérez \& Paez 1998) e em Santiago do Chile (Villegas \& Nolla 2001).

O tipo polínico Amaranthus L. alcança suas maiores quantidades na atmosfera nos meses de primavera e verão. Durante os anos de coleta apresentou uma variação na concentração anual de quase o dobro de grãos em 2001, porém as concentrações foram relativamente baixas, devido, principalmente, a plantas produtoras desse tipo polínico não serem muito abundantes na zona próxima ao captador. Hilgert (1998) registrou a presença na atmosfera desse tipo polínico apenas no mês de outubro, pois seus estudos iniciaram em maio e concluíram em novembro, com coletas alternadas de dias. As cidades de Rio de Janeiro (Oliveira Lima \& Greco 1942a), Belo Horizonte (Oliveira Lima \& Greco 1942b), Porto Alegre (Oliveira Lima et al. 1946), registraram grãos de pólen de Amaranthaceae Adans. em pequenas quantidades e em curto período. Resultados semelhantes quanto ao mês de incidência desse tipo polínico, foram encontrados no espectro polínico de Mar del Plata (Latorre \& Pérez 1997) e em La Plata 
(Nitiu \& Romero 2001), porém suas concentrações foram maiores, sendo considerado um dos principais tipos polínicos dessas localidades. Também em estudos realizados em Santiago do Chile (Villegas \& Nolla 2001), utilizando o aparelho Burkard durante um período de três anos, registrou-se concentrações máximas nos meses de fevereiro e março. No resultado do monitoramento aerobiológico daquela cidade, Amaranthus L. encontra-se entre os dez tipos polínicos mais frequientes, no caso de Caxias do Sul, foi entre os dezoito tipos polínicos.

O pólen de Rumex L. (Poligonaceae) apresentou, nestes dois anos de estudo, um comportamento principalmente primaveril, e as quantidades foram muito similares. Este tipo polínico é pouco citado em trabalhos aeropalinológicos da América do Sul (Barth 1978; Noetinger 1993).

Os demais tipos polínicos de táxons herbáceos Alternanthera Forssk., Apiaceae Lindl., Brassicaceae Burnett, Caryophyllaceae Juss., Convolvulaceae Juss. e Iridaceae Juss., não apresentaram concentrações expressivas na atmosfera da cidade.

Portanto, o pólen de Poaceae, seguido do de Cyperaceae, em suspensão na atmosfera da região Sul do Brasil, é de significativa importância em estudos aplicados e correlatos à Aeropalinologia.

\section{Agradecimentos}

À Universidad de León, pelo empréstimo do aparelho Burkard Manufacturing ${ }^{\circledR}$, o que tornou possível a realização desta pesquisa.

\section{Referências bibliográficas}

Barth, O.M. 1978. A estação polínica nas proximidades do Rio de Janeiro. Revista Brasileira de Botânica 1: 113-118.

Domínguez Vilches, E.; Galán Soldevilla, C.; Villamandos De La Torre, F. \& Infante García-Pantaleón, F. 1991. Manejo y evaluación de los datos obtenidos en los muestros aerobiológicos. Monografias de la Red Española de Aerobiologia/European Aeroallergen Network 1: 1-3.

Grant Smith, E. 1986. Sampling and identifying allergenic pollens and molds. San Antonio, Texas, Blewstone Press, 2: 1-98.

Gregory, P.H. 1973. Microbiology of the atmosphere. Inglaterra, Leonard Hill, Aylesbury, Buks.

Hilgert, S.B. 1998. Concentração polínica da Cidade de Caxias do Sul - RS, nos meses de maio a novembro de 1995 . Cadernos de Pesquisa/Universidade de Caxias do Sul 6(6): 255-276.
Hyde, H.A. \& Adams, K.F. 1958. An atlas of airborne pollen grains. New York, St. Martins Press.

Latorre, F. \& Pérez, C.F. 1997. One year of airbone pollen sampling in Mar del Plata (Argentina). Grana 36: 49-53.

Lorscheitter, M.L. \& Bernd, A.C. 1990. Estudo da chuva polínica em Porto Alegre (RS). Revista Brasileira de Alergia e Imunopatologia, edição especial, 13: 1-137.

Majas, F.D. \& Romero, E.J. 1992. Aeropalynological research in the Northeast of Buenos Aires Province, Argentina. Grana 31: 143-156.

Moore, P.D.; Webb, J.A. \& Collinson, M.E. 1991. Pollen analysis. Blackwell Scientific Publications.

Nitiu, D.S. \& Romero, E.J. 2001. Contenido polínico en la atmósfera de la ciudad de La Plata. Argentina. Pollen 11: 79-85.

Noetinger, M. 1993. Tres años de monitoreo de la lluvia polinica en la Ciudad de Buenos Aires. Archivos Argentinos de Alergia e Inmunología Clínica 24(2): 65-75.

Noetinger, M. \& Romero, E.J. 1997. Monitoreo diario y volumetrico del polen atmosferico en la ciudad de Buenos Aires. Boletín de la Sociedad Argentina de Botánica 32(3-4): 185-194.

Oliveira Lima, A. \& Greco, J.B. 1942a. Contagem de pólens atmosféricos na cidade do Rio de Janeiro. Brasil Médico 38(14-16).

Oliveira Lima, A. \& Greco, J.B. 1942b. Contagem de pólens atmosféricos na cidade de Belo Horizonte durante três anos consecutivos. Brasil - Médico 56(39): 459-461.

Oliveira Lima, A. \& Guimarães, O. 1958. Contagens de pólens na atmosfera da cidade de São Paulo. O Hospital 54: 1-879.

Oliveira Lima, A.; Greco, J.B. \& Rezende, A. 1942. Contagem de pólens aéreos na cidade de Varginha (Minas Gerais) no período de abril a junho de 1942. Brasil - Médico 56: 1-510.

Pathirane, L. 1975. Aerobiological literature in scientific periodicals. Grana 15: 145-147.

Pérez, C.F. \& Paez, M.M. 1998. Seasonal airbone pollen in Mar del Plata City, Argentina. Aerobiologia 14: 383-389.

Pire, S.M.; Anzótegui, L.M. \& Cuadrado, G.A. 1998. Flora Polínica del Nordeste Argentino. v.1. Corrientes, Argentina, Ed. Eudene.

Rosário Filho, N.A. 1983. Contagem de pólens aéreos na cidade de Curitiba. Revista da Sociedade Brasileira de Alergia e Imunopatologia 6(1-4): 12-15.

Salgado-Labouriau, M.L. 1973. Contribuição à palinologia dos Cerrados. Academia Brasileira de Ciências.

Valdés, B.; Diez, M.J. \& Fernández, I. 1987. Atlas polínico de Andalucía Occidental. Inst. de desarrollo Regional de la Universidad de Sevilla. Excma. Diputación de Cádiz. Sevilla.

Vieira, F.A.M. \& Negreiros, E.B. 1989. Arborização urbana como influência na epidemiologia da polinose na cidade de Caxias do Sul, RS, Brasil. Revista da Sociedade Brasileira de Alergia e Imunopatologia AGO: 1-114.

Villegas, G.R. \& Nolla, J.M.R. 2001. Atmospheric pollen in Santiago, Chile. Grana 40: 126-132. 